\title{
Assessment of health claims in the field of bone: a view of the Group for the Respect of Ethics and Excellence in Science (GREES)
}

\author{
O. Bruyère $\cdot$ R. Rizzoli $\cdot$ V. Coxam $•$ B. Avouac $\cdot$ \\ T. Chevalier • V. Fabien-Soulé • J. A. Kanis • \\ J.-M. Kaufman • Y. Tsouderos • J.-Y. Reginster
}

Received: 4 January 2011 / Accepted: 26 January 2011 /Published online: 25 February 2011

(C) The Author(s) 2011. This article is published with open access at Springerlink.com

\begin{abstract}
Summary Health claims for food products in Europe are permitted if the nutrient has been shown to have a beneficial nutritional or physiological effect. This paper defines health claims related to bone health and provides guidelines for the design and the methodology of clinical studies to support claims.

Introduction Regulation (EC) no. 1924/2006 on nutrition and health claims targeting food products was introduced in Europe stating that health claims shall only be permitted if the substance in respect of which the claim is made has been shown to have a beneficial nutritional or physiological effect. The objective of this paper is to define health claims related to bone health and to provide guidelines for the design and the methodology of clinical studies which need to be adopted to assert such health claims.
\end{abstract}

O. Bruyère $(\bowtie) \cdot J .-Y$. Reginster

Department of Public Health, Epidemiology and Health

Economics, University of Liège,

Liège, Belgium

e-mail: olivier.bruyere@ulg.ac.be

\section{R. Rizzoli}

Rehabilitation and Geriatrics, Geneva University Hospitals,

Geneva, Switzerland

\section{Coxam}

Department of Human Nutrition, University of Clermont Ferrand,

Clermont Ferrand, France

B. Avouac

CHU Henri Mondor,

Paris, France

\section{T. Chevalier}

Theramex,

Monte Carlo, Monaco
Methods Literature review followed by a consensus discussion during two 1-day meetings organized by the Group for the Respect of Ethics and Excellence in Science (GREES). Results The GREES identified six acceptable health claims related to bone health based on the potential of food products to show an effect on either the bioavailability of calcium or osteoclast regulatory proteins or bone turnover markers or bone mineral density or bone structure or fracture incidence. The GREES considers that welldesigned human randomized controlled trial on a relevant outcome is the best design to assess health claims. The substantiation of health claim could also be supported by animal studies showing either an improvement in bone strength with the food product or showing the relationship between changes induced by the food product on a surrogate marker and changes in bone strength.

V. Fabien-Soulé

Rousselot,

Nanterre, France

\section{J. A. Kanis}

WHO Collaborating Centre for Metabolic Bone Diseases, University of Sheffield Medical School,

Sheffield, UK

J.-M. Kaufman

Department of Endocrinology and Unit for Osteoporosis and Metabolic Bone Diseases, Ghent University Hospital,

Ghent, Belgium

Y. Tsouderos

Servier,

Paris, France 
Conclusion The consensus reached is that the level of health claim may differ according to the surrogate endpoint used and on additional animal studies provided to support the claim.

Keywords Bone $\cdot$ Health claim $\cdot$ Nutrition $\cdot$ Surrogate

\section{Introduction}

More and more food products bear health claims. The skepticism of consumers regarding functional foods is mainly due to doubts over the veracity of health claims and in the poor and often inadequate control of their claimed properties. It is important that health claims should provide genuine information to help consumers choose healthy diets. Consequently, claims should be supported by a sound and sufficient body of scientific evidence to substantiate them and be reinforced by specific consumer education.

Since health claims on food products are increasingly recognized to be important, they are being legally regulated in more and more countries around the world [1]. Although there is a general scientific consensus on how to substantiate health claims on food [2], there is no agreement on the specific approaches and indicators that can be used in different fields. Various jurisdictions have developed systematic approaches for reviewing scientific data linking food products and health with the objective of identifying the threshold of scientific evidence needed to substantiate an authoritative statement to the general public in the form of a label claim for a given marketed food product. In Europe, a regulation on nutrition and health claims made on foods was introduced in 2007. This regulation provides opportunities for the use of health claims on foods in Europe, including reduction of disease risk [3]. According to Regulation EC 1924/2006, the use of nutrition and health claims shall only be permitted if the substance in respect of which the claim is made has been shown to have a beneficial nutritional or physiological effect. A community list of permitted and rejected claims has been established and made available to the public (http://ec.europa.eu/food/ food/labellingnutrition/claims/community_register/health_ claims_en.htm).

The regulation defines a health claim in general as "any claim that states, suggests or implies that a relationship exists between a food category, a food or one of its constituents and health." All claims are addressed in Articles 13 and 14 of the Regulation EC 1924/2006 (Table 1).

In the context of health claims in foods, bone health is of potential interest as it is a major public health problem, at least in Western countries [4]. Up to $60 \%$ of the variance in bone mass is determined by genetic factors. Environmental factors account for the remainder, including nutritional intake and lifestyle habits throughout life $[5,6]$.

In the field of bone health, there are no scientifically based definitions of health claims and no uniform recommendations of the preferred study and/or methodology, even though some preparatory work had been done before the introduction of the European regulations [4]. The objective of this paper was to define the relevant biomarker for bone health and to provide recommendations for the design and the methodology of clinical studies which need to be fulfilled to assert claims related to bone health. The intent was to aid regulatory authorities in defining claims and assessing scientific evidence used to support those that relate to bone health. By establishing common criteria for these assessments, it is hoped that these recommendations will lead to harmonization of the requirements for scientific substantiation of claims worldwide.

\section{Methods}

Two 1-day meetings were organized by the Group for the Respect of Ethics and Excellence in Science (GREES). This non-for-profit organization has expertise in literature research and consensus meetings. The meetings were attended by academic scientists with expertise in the field of bone health or nutrition, members of regulatory authorities as well as industrialists with interests in health claims relating to bone. The objective of the first day of the meeting was to critically review the current literature in the field of health claims related to bone and to discuss the needs and problems to assert such claims. The objective of the second day was to reach consensus on scientifically acceptable health claims related to bone and to provide guidelines for the design and the methodology of clinical studies which need to be adopted to assert such health claims.

A literature search, using Medline database up to August 2010, was performed using keywords including health claims, nutrition, bone, osteoporosis, clinical study methodology, surrogate endpoint. A selection of relevant papers was made by $\mathrm{OB}$, RR, and JYR.

\section{Results}

The GREES panel considers that clinical data in humans are indispensable, and that health claims cannot be accepted solely on the basis of animal data. However, as discussed below, animal studies can give important information not available in humans and can provide data for the generalization of results obtained in a specific tested population to 
Table 1 Claims addressed in articles 13 and 14 of the Regulation EC 1924/2006

\begin{tabular}{|c|c|c|c|}
\hline & \multicolumn{2}{|l|}{ Article 13} & \multirow[t]{2}{*}{ Article 14} \\
\hline & Article 13.1 & Article 13.5 & \\
\hline $\begin{array}{l}\text { Referring } \\
\text { to }\end{array}$ & $\begin{array}{l}\text { the role of a nutrient or other } \\
\text { substance in growth, development } \\
\text { and the functions of the body }\end{array}$ & $\begin{array}{l}\text { the role of a nutrient or other substance in growth, } \\
\text { development and the functions of the body based on } \\
\text { newly developed scientific evidence and/or which } \\
\text { include a request for the protection of proprietary } \\
\text { data. }\end{array}$ & $\begin{array}{l}\text { the reduction of disease risk and } \\
\text { claims relating to children's } \\
\text { development and health }\end{array}$ \\
\hline $\begin{array}{l}\text { Application } \\
\text { based on }\end{array}$ & $\begin{array}{l}\text { generally accepted scientific } \\
\text { evidence }\end{array}$ & submission of an extensive scientific dossier & $\begin{array}{l}\text { submission of an extensive } \\
\text { scientific dossier }\end{array}$ \\
\hline
\end{tabular}

a larger group. Thus, different levels of heath claims should be considered based both on the endpoint used and on the information provided by animal studies.

Pre-clinical models

A variety of invasive and non-invasive techniques can be used to provide relevant endpoints [4, 7], including bioavailability studies, microarray or PCR analysis of modulated genes, histomorphometry, culture of bone forming or bone resorbing cells ex vivo, exposure to primary cell cultures to plasma harvested from treated animals, the chemistry and biochemistry of bone tissue, the assessment of biochemical indices of skeletal turnover in blood and urine, metabolic balance of calcium combined with radioactive calcium kinetics, radiogrammetry of bone radiographs, neutron activation for whole body calcium, dual x-ray absorptiometry (DXA), and the assessment of bone strength [8]. The latter endpoint is considered to be the most relevant in the field of bone health claims. Bone strength reflects both bone density and bone quality. Bone quality depends on bone architecture, mineralization, turnover, and accumulation of microdamage. Therefore, the assessment of bone health would benefit from the measurement of bone strength in vivo. No validated non-invasive tools capable of measuring bone strength in vivo are available to date. However, biomechanical tests of resistance to fracture provide an objective measure of overall bone strength. The three main types of biomechanical tests for bone strength are bending, torsional, and compression tests [9]. It is considered that both long bones and vertebral bodies should be tested since they may be differentially affected by food products.

Besides the assessment of a direct effect of the food product on bone strength, two other aims of animal data could be to better understand the mechanism of action of the food product or to validate surrogate variables used in human animal data to see if these variables reflect bone strength.
Key criteria of suitable/acceptable animal studies are:

$>$ to deliver the food product in the manner in which it will be delivered in a human setting;

$>$ to utilize a site of delivery and/or assessment site that is as closely matched as possible to the settings in which it will be used;

$>$ to utilize an animal that provides a metabolic background and physiological responsiveness comparable to humans;

$>$ to utilize a formulation of active agent that has the same composition, release, retention, and degradation properties as the formulation that will be used in humans.

Acceptable health claims in human bone health

The GREES panel considers that six different health claims could be accepted for an effect of food products on bone health. However, as already used by the European Food and Safety Authority, different wording to reflect the level of evidence of the effect could be used depending on the effect that is (always), may (demonstrated only under certain circumstances) or might be (logically expected benefit from physiology but yet not demonstrated) beneficial for bone health.

1. Improvement of calcium bioavailability

Calcium bioavailability may be defined as the proportion of calcium in foods which is absorbed and utilized for normal metabolic functions. In addition to the amount of calcium in the diet, the fractional absorption of dietary calcium in food and its retention in the body are also a factor that determines the availability of calcium for bone development and maintenance of bone health $[10,11]$. Many methods can be used to assess bioavailability (i.e., classical and isotopic balances, urinary excretion, isotope labeling in the urine, plasma, and bones) [12]. The group considers that an increase in bioavailability is not 
beneficial if not accompanied by calcium retention in the body. A food product with an effect on calcium bioavailability with or without calcium retention data, unless associated with appropriate animal studies would not fulfill a claim related to article 14 . However, food products that show an effect on bioavailability and calcium retention could have an article 13 claim: "X increases calcium absorption" or "X increases calcium bioavailability".

2. Maintenance of bone metabolism (through an effect on osteoclast regulatory proteins)

The transition of osteoclast precursors to mature osteoclasts that are capable of resorbing bone is tightly regulated by osteoclast regulatory proteins that either affect the differentiation and proliferation of osteoclast precursors into mature osteoclasts or are involved in the coupling between osteoblasts and osteoclasts [13]. Markers of osteoclastogenesis include receptor activator of nuclear factor kappa-B ligand and osteoprotegerin, whereas markers of osteoclast number include tartrate-resistant acid phosphatase and cathepsin K. These proteins contribute to bone metabolism but are not yet strongly associated with elements of bone strength. A food product with an effect on osteoclast regulatory proteins, unless supported by animal studies (see next BMD and BTM sections) would not fulfill a claim related to article 14 . The product, however, might have the label under the article 13: "X contributes to the maintenance of bone metabolism".

3. Maintenance or changes in bone turnover marker

A determinant of bone strength that is not assessed by bone mineral density (BMD) is the rate of bone remodeling. Depending upon their origin, bone turnover markers (BTMs) are classified as indices of bone resorption or formation [14-17]. The rate of bone resorption and formation can be estimated by assays that measure the serum concentration or urinary excretion of different target molecules specific to these cellular processes. GREES panel recommends the inclusion of reference markers of bone formation (serum procollagen type I N propeptide, s-PINP) and resorption (serum $\mathrm{C}$-terminal cross-linking telopeptide of type I collagen, s-CTX) in keeping with the recommendations of the International Osteoporosis Foundation [18]. A food product with a positive BTM balance might have the claim: "X maintains normal bone remodeling that could contribute to the normal structure and function of bones" or " $\mathrm{X}$ increases markers of bone formation that could contribute to the normal structure and function of bones" or "X decreases markers of bone resorption that could contribute to the normal structure and function of bones".
As in the case of BMD, BTMs are only indicators of fracture risk, but the change in BTM induced by a product is not necessarily associated with a change in fracture risk or bone strength. In this regard, animal models are useful to assess if changes in BTMs due to the intake of the food product are associated with an increase in bone strength. A food product with an effect on BTMs together with animal studies that showed improved bone strength or a relationship between changes in BTMs induced by the food product and bone strength could have the claim: " $\mathrm{X}$ contributes to the maintenance of normal bone remodeling (or increases bone formation or decreases bone resorption) that is associated with bone strength" or "X contributes to the maintenance of normal bone remodeling (or increases bone formation or decreases bone resorption) that increases bone strength" or " $\mathrm{X}$ increases bone strength".

4. Maintenance or improvement in bone structure

The key role of bone microarchitecture in bone health was suggested by the classic definition of osteoporosis adopted in 1993 [19]. Methods for investigating 3-D bone microarchitecture and bone strength include in vitro $\mu \mathrm{CT}$, in vitro $\mu \mathrm{MRI}$, in vivo pQCT, and in vivo high-resolution MRI [20]. They aim to quantify various determinants of bone strength such as bone geometry, volumetric bone density, microarchitecture, and properties of the bone matrix [21]. A food product with an effect on bone microarchitecture could have the claim: " $\mathrm{X}$ improves (or maintains) bone microarchitecture that could contribute to the normal structure and function of bones".

It is considered that the assessment of bone structure with the tools currently available in man is not sufficiently validated to be a reliable surrogate of bone strength. For this reason, animal models are needed to assess the relationship between changes in bone microarchitecture induced by the food product and any increase in bone strength. A food product with an effect on microarchitecture of the human bone and animals studies that show improvement in bone strength or show the relationship between change in bone structure induced by the food product and bone strength could have the claim: " $\mathrm{X}$ improves bone microarchitecture that increases bone strength" or "X increases bone strength"

5. Maintenance or increase in bone mineral density

Bone strength is determined by many factors, including bone mass. Bone mass is estimated in clinical practice by the measurement of BMD. BMD, as measured by DXA, represents an estimate of the quantity of mineral (grams of calcium) divided by the two-dimensional area of the bone [22]. There is a strong relationship between the risk of fracture and 
BMD but there is a wide overlap in the bone densities of patients who develop a fracture and those who do not. Since BMD is only a surrogate marker for bone strength or fracture risk, and since product-induced changes in BMD are not clearly associated with changes in bone strength or fracture risk, an increase in BMD may not be associated with an increased bone strength or decreased fracture risk [23]. A food product with a positive effect on BMD could have the claim: " $\mathrm{X}$ increases BMD. A low BMD is associated with an increased risk of fracture" or "X maintains BMD. A low BMD is associated with an increased risk of fracture".

Animal models are appropriate to determine whether an increase in BMD associated with a food product is accompanied by an increase in bone strength. A food product with a positive effect on BMD, together with animal studies showing an improvement in bone strength or showing a relationship between BMD changes induced by the food product and bone strength, could have the claim: " $\mathrm{X}$ increases (or maintains) BMD that could reduce the risk of fracture" or " $\mathrm{X}$ increases (or maintains) BMD that increases bone strength" or " $\mathrm{X}$ increases bone strength".

6. Reduction of the risk of fracture

A reduction of the incidence of fracture is a major aim of food products beneficial to skeletal health, but according to the regulation cannot be claimed as such without mentioning the effect on a risk factor. However, a reduction in the fracture risk is obviously supportive for a claim on the reduction of an identified risk factor. For non-spinal fractures, either femoral (hip) or major non-vertebral (pelvis, distal femur, proximal tibia, ribs, proximal humerus forearm, and hip) fractures should be assessed.

\section{Design of clinical studies}

\section{Population}

The subjects studied should be representative of the population targeted for the food product. The applicant should take all necessary precautions to make sure that the tested population is equivalent to the user population with respect of ethnicity, age, physiological status (such as menopause for example), life habits (such as exercise) and diet. No densitometric criteria are required for inclusion. However, the experimental and the control group must show no significant differences in term of baseline BMD.

2. Design

The ideal design would be a multicentre randomized controlled study (RCT). The control could be a placebo, another active product or nothing, depending on the tested food. When possible, subjects and/or investigators should be blinded of the intervention. Treatment and control groups should be balanced with respect to gender, age, menopausal status, dietary habits, or underlying diseases. The GREES panel recognizes that a RCT is not always possible in practice or from an ethical point of view. Since the totality of the evidence should be weighed for the substantiation of a claim, well-designed prospective cohort studies, case-control studies and/or observational studies of high quality could be acceptable if accompanied by other data (e.g., animal data, effect on multiple surrogate endpoints). Cross-over studies design can also be considered. All the efforts should be made to eliminate potential confounders.

3. Duration of study

The duration of the trial should be predetermined and should depend on the outcome. For BMD, duration of at least 1 year seems necessary. For BTMs, a 3month study is the minimum. The primary efficacy endpoint should be assessed at the end of the predetermined treatment period in comparison with the measurement at baseline. Intermediate measurements are also recommended.

4. Statistical analysis

Intention-to-treat analysis should be the primary method of evaluation. Statistical significance will be inferred if a $P$ value is equal to or less than 0.05 . The beta risk will be equal to or less than $20 \%$. The sample size of the study must be calculated prior to the start of the study. Possible confounding variables should be managed using appropriate statistical analysis. Within group (end vs. baseline) and between groups comparisons should be made.

5. Diet habit and lifestyle

The control of critical effect modifiers such as physical activity, synergies with a multitude of other nutrients and the influence of nutrigenomic relationships must be taken into account.

Intakes of other nutrients or foods, on which the tested nutrient is dependent, must be optimized. Any supplementation with other food products known to have an effect on bone (e.g., calcium and/or vitamin D) should be consistent within all patient groups.

6. Observance

Observance of food product intake should be monitored during the study to be able to perform preplanned analyses on individuals with high and poor compliance rates or analyses of dose-response.

7. Safety

All adverse experiences occurring during the course of clinical trials should be fully documented with separate analysis of adverse events, dropouts, and patients who died while being on the study. 


\section{Conclusion}

According to the European regulation, the use of nutrition and health claims shall only be permitted if the food product has been shown to have a beneficial nutritional or physiological effect in agreement with the health claim. However, it must also be pointed out that during the evaluation of the health claim, besides the characterization of the effect, important elements will be taken into account, such as the characterization of the food and the substantiation of the effect. In the field of bone health, claimed effects are not sufficiently defined and there are no standardized recommendations for the design and the methodology of clinical studies needed to reach such health claims. The consensus reached by the GREES is that the level of health claim may differ according to the surrogate endpoint used and on additional animal studies provided to support the claim. The ideal study design is a RCT but, is some particular cases, prospective cohort, case-control, or observational studies can be acceptable. In our opinion, general principles of the consensus reached are in line with the principles adopted in the EFSA's published opinions. This consensus is subject to future modifications when new validated surrogate markers will be available.

Acknowledgment The authors would like to thank Professor Ambroise Martin, from University Claude Bernard in Lyon, France, and member of the NDA panel of the EFSA, for participation in the meetings.

Conflicts of interest $\mathrm{O}$ Bruyere receives grants or has been reimbursed for attending meetings from GlaxoSmithKline, IBSA, MSD, Novartis, Rottapharm, Servier, Theramex and Wyeth. He also gives advice to the European Food Safety Authority and the French Food Safety Agency. R Rizzoli is at the Speaker Bureau of Amgen, GSK, Merck, Novartis, Nycomed, Roche, and Servier. He is a member of the Scientific Advisory Boards of Amgen, Danone, Eli Lilly, Novartis, Nycomed, Roche, and Servier; and editor of Bone and Associate Editor of Osteoporosis International. He is treasurer and member of the Executive Committee of the International Osteoporosis Foundation. V Coxam receives grants from Danone, Greentech, Lesieur, Rousselot and Servier. B Avouac received fees from Servier, Novartis, Negma, Amgen, GlaxoSmithKline, Roche, Nycomed, Theramex, UCB, Expanscience, Lundbeck, Janssen Cilag and Horus. JA Kanis consults for a large number of companies and receives grants or gives advice to nongovernmental agencies. JM Kaufman received fees as consultant or received financial support for attending meetings from Amgen, Eli Lilly, GlaxoSmithKline, Merck Sharpe \& Dohme, Novartis, Novo Nordisk, Nycomed, Procter \& Gamble, Roche, Sanofi-Aventis, Servier, and Wyeth. He received grant support from GlaxoSmithKline, Merck Sharpe \& Dohme, Novartis, Roche, and the Flemish Fund for Scientific Research. $\mathrm{He}$ is a (alternate) member of a commission on drug reimbursement with the Belgian health authorities. J-Y Reginster has received consulting fees or payments for participating in advisory boards for Servier, Novartis, Negma, Lilly, Wyeth, Amgen, GlaxoSmithKline, Roche, Merckle, Nycomed, NPS, Theramex, and $\mathrm{UCB}$. He has received lecture fees when speaking at the invitation of Merck Sharp and Dohme, Lilly, Rottapharm, IBSA, Genevrier,
Novartis, Servier, Roche, GlaxoSmithKline, Teijin, Teva, Ebewee Pharma, Zodiac, Analis, Theramex, Nycomed, and Novo Nordisk; and grant support from Bristol Myers Squibb, Merck Sharp \& Dohme, Rottapharm, Teva, Lilly, Novartis, Roche, GlaxoSmithKline, Amgen, and Servier.

Open Access This article is distributed under the terms of the Creative Commons Attribution Noncommercial License which permits any noncommercial use, distribution, and reproduction in any medium, provided the original author(s) and source are credited.

\section{References}

1. Jones PJ, Asp NG, Silva P (2008) Evidence for health claims on foods: how much is enough? Introduction and general remarks. J Nutr 138:1189S-1191S

2. Grossklaus R (2009) Codex recommendations on the scientific basis of health claims. Eur J Nutr 48(Suppl 1):S15-S22

3. Asp NG, Bryngelsson S (2008) Health claims in Europe: new legislation and PASSCLAIM for substantiation. J Nutr 138:1210S$1215 \mathrm{~S}$

4. Prentice A, Bonjour JP, Branca F, Cooper C, Flynn A, Garabedian M, Muller D, Pannemans D, Weber P (2003) PASSCLAIMbone health and osteoporosis. Eur J Nutr 42(Suppl 1):I28-I49

5. Rizzoli R (2008) Nutrition: its role in bone health. Best Pract Res Clin Endocrinol Metab 22:813-829

6. Rizzoli R, Bianchi ML, Garabedian M, McKay HA, Moreno LA (2010) Maximizing bone mineral mass gain during growth for the prevention of fractures in the adolescents and the elderly. Bone 46:294-305

7. Bonjour JP, Ammann P, Rizzoli R (1999) Importance of preclinical studies in the development of drugs for treatment of osteoporosis: a review related to the 1998 WHO guidelines. Osteoporos Int 9:379-393

8. Muschler GF, Raut VP, Patterson TE, Wenke JC, Hollinger JO (2010) The design and use of animal models for translational research in bone tissue engineering and regenerative medicine. Tissue Eng Part B Rev 16:123-145

9. Ammann P (2009) Bone strength and ultrastructure. Osteoporos Int 20:1081-1083

10. Cashman KD (2002) Calcium intake, calcium bioavailability and bone health. Br J Nutr 87(Suppl 2):S169-S177

11. Fairweather-Tait SJ, Teucher B (2002) Calcium bioavailability in relation to bone health. Int J Vitam Nutr Res 72:13-18

12. Gueguen L, Pointillart A (2000) The bioavailability of dietary calcium. J Am Coll Nutr 19:119S-136S

13. Leeming DJ, Alexandersen P, Karsdal MA, Qvist P, Schaller S, Tanko LB (2006) An update on biomarkers of bone turnover and their utility in biomedical research and clinical practice. Eur J Clin Pharmacol 62:781-792

14. Civitelli R, Armamento-Villareal R, Napoli N (2009) Bone turnover markers: understanding their value in clinical trials and clinical practice. Osteoporos Int 20:843-851

15. Brown JP, Albert C, Nassar BA, Adachi JD, Cole D, Davison KS, Dooley KC, Don-Wauchope A, Douville P, Hanley DA, Jamal SA, Josse R, Kaiser S, Krahn J, Krause R, Kremer R, Lepage R, Letendre E, Morin S, Ooi DS, Papaioaonnou A, Ste-Marie LG (2009) Bone turnover markers in the management of postmenopausal osteoporosis. Clin Biochem 42:929-942

16. Vasikaran SD (2008) Utility of biochemical markers of bone turnover and bone mineral density in management of osteoporosis. Crit Rev Clin Lab Sci 45:221-258 
17. Vasikaran SD, Glendenning P, Morris HA (2006) The role of biochemical markers of bone turnover in osteoporosis management in clinical practice. Clin Biochem Rev 27:119-121

18. Vasikaran SD, Eastell R, Bruyere O, Foldes AJ, Garnero P, Griesmacher A, McClung M, Morris HA, Silverman S, Trenti T, Wahl DA, Cooper C, Kanis JK (2011) Markers of bone turnover for the prediction of fracture risk and monitoring of osteoporosis treatment: a need for international reference standards. Osteoporos Int 22(2):391-420

19. Consensus development conference (1993) Diagnosis, prophylaxis, and treatment of osteoporosis. Am J Med 94:646-650
20. Lespessailles E, Chappard C, Bonnet N, Benhamou CL (2006) Imaging techniques for evaluating bone microarchitecture. Joint Bone Spine 73:254-261

21. Brandi ML (2009) Microarchitecture, the key to bone quality. Rheumatol Oxf 48(Suppl 4):iv3-iv8

22. Hochberg MC (2006) Recommendations for measurement of bone mineral density and identifying persons to be treated for osteoporosis. Rheum Dis Clin North Am 32:681-689

23. Seeman E (2007) Is a change in bone mineral density a sensitive and specific surrogate of anti-fracture efficacy? Bone $41: 308-317$ 